\title{
On Representations of Conformal Field Theories and the Construction of Orbifolds
}

\author{
P.S. Montague * \\ Department of Applied Mathematics and Theoretical Physics \\ University of Cambridge \\ Silver Street \\ Cambridge CB3 9EW \\ U.K.
}

May 10, 2018

\begin{abstract}
We consider representations of meromorphic bosonic chiral conformal field theories, and demonstrate that such a representation is completely specified by a state within the theory. The necessary and sufficient conditions upon this state are derived, and, because of their form, we show that we may extend the representation to a representation of a suitable larger conformal field theory. In particular, we apply this procedure to the lattice (FKS) conformal field theories, and deduce that Dong's proof of the uniqueness of the twisted representation for the reflection-twisted projection of the Leech lattice conformal field theory generalises to an arbitrary even (self-dual) lattice. As a consequence, we see that the reflection-twisted lattice theories of [1] are truly self-dual, extending the analogies with the theories of lattices and codes which were being pursued. Some comments are also made on the general concept of the definition of an orbifold of a conformal field theory in relation to this point of view.
\end{abstract}

\footnotetext{
${ }^{1}$ January-December 1995: visiting Department of Physics and Mathematical Physics, University of Adelaide, Adelaide, SA 5005, Australia
} 


\section{Introduction}

In this letter, we shall study only meromorphic bosonic conformal field theories. The classification, in particular, of the self-dual theories has been a major goal in recent years, partly because of its physical relevance to heterotic string theory [9], but also because of its intrinsic interest, particularly in view of the surprising links to the Monster group [5]. In [1, 3] these links were extended as part of a program of studying the apparent and, as it turned out, deep connections and analogies between bosonic conformal field theories and the theories of lattices and codes. One aspect of the work of [1] and similarly [6] is the attempt to systematise and axiomatise the study of these theories. In the spirit of this approach, we study here in section 2 the representations of such conformal field theories, showing that they may be described entirely within the original theory in terms of a certain corresponding state, and as a consequence of this point of view we present, in section 3, a technique by which one may extend a representation of a conformal field theory to a theory in which it is suitably embedded and hence we tighten up the analogies between lattices and conformal field theories set out in [3] by demonstrating that the reflection-twisted orbifolds of the FKS lattice conformal field theories are self-dual in the sense of being their own unique meromorphic representation rather than simply requiring that their partition function be modular invariant. Finally, in section $₫$, we present some comments and conclusions.

\section{Representations of Meromorphic Bosonic Confor- mal Field Theories}

We take $\mathcal{H}$ to be a meromorphic bosonic conformal field theory with vertex operators denoted by $V(\psi, z), \psi \in \mathcal{H}$, and consider a representation of $\mathcal{H}$ given by a set of vertex operators $U$ and a Hilbert space $\mathcal{K}$ (see [1] for a precise definition of these concepts), i.e.

$$
U(\psi, z) U(\phi, w)=U(V(\psi, z-w) \phi, w)
$$

for $\psi, \phi \in \mathcal{H}$, and also $U(|0\rangle, z) \equiv 1$ (where $|0\rangle$ denotes the vacuum state in $\mathcal{H}$ - the unique state invariant under the $\mathrm{SU}(1,1)$ symmetry generated by the Virasoro modes $L_{0}$, $\left.L_{ \pm 1}\right)$.

Now, the existence of the representation is equivalent to the existence of intertwining operators [1] $W(\chi, z), \chi \in K$, defined by $W(\chi, z) \psi=e^{z L-1} U(\psi,-z) \chi$, such that we have the "intertwining" locality relation

$$
U(\psi, z) W(\chi, w)=W(\chi, w) V(\psi, z)
$$


where the left and right hand sides of the relation are strictly only defined for $|z|>|w|$ and $|w|>|z|$ respectively, and the equality is taken to mean that when we take matrix elements of both sides in the appropriate Hilbert spaces the functions that are so obtained on either side may be analytically continued into one another.

We shall also require that our conformal field theory $\mathcal{H}$ possess a hermitian structure [1], by which we mean that there exists an antilinear map on $\psi \mapsto \bar{\psi}$ on $\mathcal{H}$ such that

$$
V(\bar{\psi}, z)=V\left(e^{z^{*} L_{1}} z^{*-2 L_{0}} \psi, 1 / z^{*}\right)^{\dagger}
$$

and we shall say that the representation is hermitian if a similar relation holds for the vertex operators $U$ for some antilinear map on $\mathcal{K}$.

Consider the matrix element

$$
\left\langle\chi\left|U\left(\psi_{1}, z_{1}\right) U\left(\psi_{2}, z_{2}\right) \ldots U\left(\psi_{n}, z_{n}\right)\right| \chi\right\rangle
$$

for some quasi-primary (i.e. annihilated by $L_{1}$ ) state $\chi \in \mathcal{K}$ and states $\psi_{1}, \ldots \psi_{n} \in \mathcal{H}$. Using the definition of the intertwining operators, this becomes

$$
\left\langle\chi\left|U\left(\psi_{1}, z_{1}\right) U\left(\psi_{2}, z_{2}\right) \ldots U\left(\psi_{n-1}, z_{n-1}\right) e^{z_{n} L_{-1}} W\left(\chi,-z_{n}\right)\right| \psi_{n}\right\rangle
$$

and then using the fact that $L_{-1}$ is the generator of translations we obtain

$$
\left\langle\chi\left|U\left(\psi_{1}, z_{1}-z_{n}\right) U\left(\psi_{2}, z_{2}-z_{n}\right) \ldots U\left(\psi_{n-1}, z_{n-1}-z_{n}\right) W\left(\chi,-z_{n}\right)\right| \psi_{n}\right\rangle
$$

and thus the intertwining locality relation gives

$$
\begin{array}{rll}
\langle\chi| U\left(\psi_{1}, z_{1}\right) U\left(\psi_{2}, z_{2}\right) & \ldots & U\left(\psi_{n}, z_{n}\right)|\chi\rangle= \\
\left\langle P\left(z_{n}^{*}\right)\right| V\left(\psi_{1}, z_{1}-z_{n}\right) V\left(\psi_{2}, z_{2}-z_{n}\right) & \ldots & V\left(\psi_{n-1}, z_{n-1}-z_{n}\right)\left|\psi_{n}\right\rangle
\end{array}
$$

where

$$
\left\langle P\left(z^{*}\right)\right| \equiv\langle\chi| W(\chi,-z) .
$$

Now, in view of this, let us start again, and take (7) as defining an abstract representation described by vertex operators $U$ of the conformal field theory $\mathcal{H}$. We shall investigate what properties the now unknown state $P(z) \equiv \sum_{n \geq 0} P_{n} z^{-n}\left(P_{n}\right.$ of conformal weight $n$ ) must satisfy in order that this be a representation. This representation is built up on the state $\chi$ by the action of the $U$ 's. Note that, in order to make sense of this in Hilbert space terms, we shall require that the representation be hermitian, so that the interpretation in terms of matrix elements is simplified. There is a vast degeneracy in this notation, and in order to identify the Hilbert space behind the representation one must quotient out by 
all the null states (identified using (ㅁ) ). Any technical problems to do with separability of the resulting space will not be addressed here, though in any given case it will be easy to identify that there is actually a finite number of states at each conformal weight.

Let us first consider the representation property (11) and impose that (7) satisfy this. There are two classes of cases to consider - ones involving $\psi_{n}$ and ones not. For the first case:

$$
\begin{aligned}
& \left\langle\chi\left|U\left(\psi_{1}, z_{1}\right) \ldots U\left(\psi_{i}, z_{i}\right) U\left(\psi_{i+1}, z_{i+1}\right) \ldots U\left(\psi_{n}, z_{n}\right)\right| \chi\right\rangle \\
= & \left\langle P\left(z_{n}^{*}\right)\left|V\left(\psi_{1}, z_{1}-z_{n}\right) \ldots V\left(\psi_{i}, z_{i}-z_{n}\right) V\left(\psi_{i+1}, z_{i+1}-z_{n}\right) \ldots V\left(\psi_{n-1}, z_{n-1}-z_{n}\right)\right| \psi_{n}\right\rangle \\
= & \left\langle P\left(z_{n}^{*}\right)\left|V\left(\psi_{1}, z_{1}-z_{n}\right) \ldots V\left(V\left(\psi_{i}, z_{i}-z_{i+1}\right) \psi_{i+1}, z_{i+1}-z_{n}\right) \ldots V\left(\psi_{n-1}, z_{n-1}-z_{n}\right)\right| \psi_{n}\right\rangle \\
= & \left\langle\chi\left|U\left(\psi_{1}, z_{1}\right) \ldots U\left(V\left(\psi_{i}, z_{i}-z_{i+1}\right) \psi_{i+1}, z_{i+1}\right) \ldots U\left(\psi_{n}, z_{n}\right)\right| \chi\right\rangle
\end{aligned}
$$

and for the second case:

$$
\begin{aligned}
& \left\langle\chi\left|U\left(\psi_{1}, z_{1}\right) \ldots U\left(\psi_{n}, z_{n}\right)\right| \chi\right\rangle \\
= & \left\langle P\left(z_{n}^{*}\right)\left|V\left(\psi_{1}, z_{1}-z_{n}\right) \ldots V\left(\psi_{n-1}, z_{n-1}-z_{n}\right)\right| \psi_{n}\right\rangle \\
= & \left\langle\chi\left|U\left(\psi_{1}, z_{1}\right) \ldots U\left(V\left(\psi_{n-1}, z_{n-1}-z_{n}\right) \psi_{n}, z_{n}\right)\right| \chi\right\rangle .
\end{aligned}
$$

Now, $U(|0\rangle, z)=1$ is clearly consistent for $\psi_{i}=|0\rangle$ with $i \neq n$. For $i=n$, there are two cases $-n=1$ and $n>1$.

$n=1$ :

$$
\left\langle P\left(z^{*}\right) \mid 0\right\rangle=1
$$

$n>1:$

$$
\begin{aligned}
& \left\langle P\left(z_{n}^{*}\right)\left|V\left(\psi_{1}, z_{1}-z_{n}\right) \ldots V\left(\psi_{n-1}, z_{n-1}-z_{n}\right)\right| 0\right\rangle \\
= & \left\langle P\left(z_{n}^{*}\right)\left|V\left(\psi_{1}, z_{1}-z_{n}\right) \ldots e^{\left(z_{n-1}-z_{n}\right) L_{-1}}\right| \psi_{n-1}\right\rangle \\
= & \left\langle P\left(z_{n}^{*}\right)\left|e^{\left(z_{n-1}-z_{n}\right) L_{-1}} V\left(\psi_{1}, z_{1}-z_{n-1}\right) \ldots\right| \psi_{n-1}\right\rangle .
\end{aligned}
$$

Hence, we require

$$
\left\langle P\left(z^{*}\right)\right|=\left\langle P\left(w^{*}\right)\right| e^{(z-w) L_{-1}} .
$$

[Note that this is simply the usual $L_{-1}$ commutation relation for vertex operators when we rewrite it in terms of $W$ using (8).]

Now let us consider the hermitian structure of the representation. Consider

$$
\begin{aligned}
& \left\langle\chi\left|U\left(e^{z_{n}^{*} L_{1}} z_{n}^{*-2 L_{0}} \overline{\psi_{n}}, 1 / z_{n}^{*}\right) \ldots\right| \chi\right\rangle^{*} \\
= & \left\langle\overline{\psi_{1}}\left|z_{1}{ }^{-2 L_{0}} e^{z_{1} L_{-1}} V^{\dagger}\left(e^{z_{2}^{*} L_{1}} z_{2}^{*-2 L_{0}} \overline{\psi_{2}}, \frac{1}{z_{2}^{*}}-\frac{1}{z_{1}^{*}}\right) \ldots\right| P\left(1 / z_{1}\right)\right\rangle
\end{aligned}
$$




$$
\begin{aligned}
& =\left\langle\overline{\psi_{1}}\left|z_{1}{ }^{-2 L_{0}} e^{z_{1} L_{-1}} V\left(e^{\left(\frac{1}{z_{2}}-\frac{1}{z_{1}}\right) L_{1}}\left(\frac{1}{z_{2}}-\frac{1}{z_{1}}\right)^{-2 L_{0}} e^{-z_{2} L_{1}} z_{2}{ }^{-2 L_{0}} \psi_{2}, \frac{z_{1} z_{2}}{z_{1}-z_{2}}\right) \ldots\right| P\left(1 / z_{1}\right)\right\rangle \\
& =\left\langle\overline{\psi_{1}}\left|V\left(e^{\left(z_{1}-z_{2}\right) L_{1}}\left(z_{1}-z_{2}\right)^{-2 L_{0}} \psi_{2}, \frac{1}{z_{1}-z_{2}}\right) \ldots z_{1}^{-2 L_{0}} e^{z_{1} L_{-1}}\right| P\left(1 / z_{1}\right)\right\rangle \\
& =\left\langle P\left(1 / z_{1}\right)\left|e^{z_{1}^{*} L_{1}} z_{1}^{*-2 L_{0}} V\left(\overline{\psi_{n}}, z_{1}^{*}-z_{n}^{*}\right) \ldots\right| \overline{\psi_{1}}\right\rangle^{*} .
\end{aligned}
$$

We require this, for hermiticity, to be equal to

$$
\left\langle P\left(z_{1}^{*}\right)\left|V\left(\psi_{n}, z_{n}-z_{1}\right) \ldots\right| \psi_{1}\right\rangle
$$

using locality in the representation to reorder the operators as appropriate. This is, using the reality of the underlying conformal field theory (see below),

$$
\left\langle\overline{P\left(z_{1}^{*}\right)}\left|V\left(\overline{\psi_{n}}, z_{1}^{*}-z_{n}^{*}\right) \ldots\right| \overline{\psi_{1}}\right\rangle^{*}
$$

Hence, we have a hermitian structure in the representation if

$$
\langle\overline{P(z)}|=\left\langle P\left(1 / z^{*}\right)\right| e^{z L_{1}} z^{-2 L_{0}} .
$$

A further property which we may wish to impose on the representation is that of reality. The representation is said to be real if there is an antilinear map $\rho \mapsto \bar{\rho}$ on $\mathcal{K}$ such that $\overline{\bar{\rho}}=\rho, L_{-1} \bar{\rho}=-\overline{L_{-1} \rho}$ and

$$
\left(f_{\rho_{1} \phi \rho_{2}}\right)^{*}=(-1)^{h_{1}+h_{\phi}+h_{2}} f_{\bar{\rho}_{1} \bar{\phi} \bar{\rho}_{2}}
$$

where $L_{0} \rho_{j}=h_{j} \rho_{j}$ for $j=1,2, L_{0} \phi=h_{\phi} \phi$ and $f_{\rho_{1} \phi \rho_{2}}=\left\langle\bar{\rho}_{1}|U(\phi, 1)| \rho_{2}\right\rangle$. Also, we require that if $L_{0} \rho=h_{\rho} \rho$ then $L_{0} \bar{\rho}=h_{\rho} \bar{\rho}$. An analogous result holds true for $\mathcal{H}$ [1] simply as a consequence of its hermitian structure (as we made use of above). Reality for the representation is a necessary requirement if we wish to endow $\mathcal{H} \oplus \mathcal{K}$ with the structure of a conformal field theory [1], i.e. it is relevant in the construction of $\mathbf{Z}_{2}$-orbifold theories.

Using the required structure as a paradigm, we define

$$
\overline{U(\psi, z) \chi}=U\left(\bar{\psi},-z^{*}\right) \chi
$$

(Note that we are implicitly taking the state $\chi$ to be real.) This trivially satisfies the requirements of antilinearity, $\overline{\bar{\rho}}=\rho, L_{-1} \bar{\rho}=-\overline{L_{-1} \rho}$ and $L_{0} \bar{\rho}=h_{\rho} \bar{\rho}$, since (denoting the state corresponding to the Virasoro modes as $\psi_{L}\left(=\overline{\psi_{L}}\right)$, i.e. $\left.V\left(\psi_{L}, z\right) \equiv \sum_{n} L_{n} z^{-n-2}\right)$

$$
\overline{U\left(\psi_{L}, z\right) U(\psi, w) \chi}=\overline{U\left(V\left(\psi_{L}, z-w\right) \psi, w\right) \chi}
$$




$$
\begin{aligned}
& =U\left(\overline{V\left(\psi_{L}, z-w\right) \psi},-w^{*}\right) \chi \\
& =U\left(V\left(\psi_{L}, w^{*}-z^{*}\right) \bar{\psi},-w^{*}\right) \chi \\
& =U\left(\psi_{L},-z^{*}\right) U\left(\bar{\psi},-w^{*}\right) \chi \\
& =U\left(\psi_{L},-z^{*}\right) \overline{U(\psi, w) \chi} .
\end{aligned}
$$

Finally, in order to satisfy (18), we require

$$
\left\langle\rho_{2}\left|U^{\dagger}(\phi, 1)\right| \overline{\rho_{1}}\right\rangle=\left\langle\rho_{1}|U(\bar{\phi},-1)| \overline{\rho_{2}}\right\rangle,
$$

or

$$
\left\langle\rho_{2}\left|U\left(e^{L_{1}} \bar{\phi}, 1\right)\right| \overline{\rho_{1}}\right\rangle=\left\langle\rho_{1}|U(\bar{\phi},-1)| \overline{\rho_{2}}\right\rangle,
$$

i.e.

$$
\langle\rho| U^{\dagger}\left(\psi_{2}, z_{2}\right) U\left(e^{L_{1}} \bar{\phi}, 1\right) \overline{U\left(\psi_{1}, z_{1}\right)|\chi\rangle}=\langle\chi| U^{\dagger}\left(\psi_{1}, z_{1}\right) U(\bar{\phi},-1) \overline{U\left(\psi_{2}, z_{2}\right)|\chi\rangle} .
$$

Thus, we require

$$
\begin{aligned}
& \langle\chi| U\left(e^{z_{2}^{*} L_{1}} z_{2}^{*-2 L_{0}} \overline{\psi_{2}}, 1 / z_{2}^{*}\right) U\left(e^{L_{1}} \bar{\phi}, 1\right) U\left(\overline{\psi_{1}},-z_{1}^{*}|\chi\rangle\right. \\
= & \left\langle\chi\left|U\left(e^{z_{1}^{*} L_{1}} z_{1}^{*-2 L_{0}} \overline{\psi_{1}}, 1 / z_{1}^{*}\right) U(\bar{\phi},-1) U\left(\overline{\psi_{2}},-z_{2}^{*}\right)\right| \chi .\right.
\end{aligned}
$$

The right hand side is, by definition,

$$
\left\langle P\left(-z_{2}\right)\left|V\left(e^{z_{1}^{*} L_{1}} z_{1}^{*-2 L_{0}} \overline{\psi_{1}}, 1 / z_{1}^{*}+z_{2}^{*}\right) V\left(\bar{\phi},-1+z_{2}^{*}\right)\right| \overline{\psi_{2}}\right\rangle,
$$

while the left hand side is

$$
\begin{aligned}
& \left\langle\chi\left|U\left(e^{-z_{1} L_{1}} z_{1}{ }^{-2 L_{0}} \psi_{1},-1 / z_{1}\right) U(\phi, 1) U\left(\psi_{2}, z_{2}\right)\right| \chi\right\rangle \\
= & \left\langle P\left(z_{2}^{*}\right)\left|V\left(e^{-z_{1} L_{1}} z_{1}{ }^{-2 L_{0}} \psi_{1},-1 / z_{1}-z_{2}\right) V\left(\phi, 1-z_{2}\right)\right| \psi_{2}\right\rangle .
\end{aligned}
$$

So, we see again from reality of the underlying conformal field theory that we have a real representation if

$$
\langle\overline{P(z)}|=\left\langle P\left(-z^{*}\right)\right| .
$$

Collecting together the above results, we see that (7) defines a hermitian representation of $\mathcal{H}$ if

$$
\begin{aligned}
\left\langle P\left(z^{*}\right) \mid 0\right\rangle & =1 \\
\left\langle P\left(z^{*}\right)\right| & =\left\langle P\left(w^{*}\right)\right| e^{(z-w) L_{-1}} \\
\langle\overline{P(z)}| & =\left\langle P\left(1 / z^{*}\right)\right| e^{z L_{1}} z^{-2 L_{0}} .
\end{aligned}
$$


If, further, we require this representation to be real then we may replace (30) by

$$
\begin{aligned}
\langle\overline{P(z)}| & =\left\langle P\left(-z^{*}\right)\right| \\
\left\langle P\left(-z^{*}\right)\right| & =\left\langle P\left(1 / z^{*}\right)\right| e^{z L_{1}} z^{-2 L_{0}},
\end{aligned}
$$

where (32) is the usual skew-symmetry relation on the vertex operators [6].

Thus, any given representation of a conformal field theory is completely specified by a state within that theory (knowledge of $\langle P(1)|$ is clearly sufficient), and no explicit form for the vertex operators acting in the representation space need be considered.

\section{Extension of a Representation: An Application to FKS Lattice Conformal Field Theories}

Let $\Lambda$ be an even self-dual lattice of dimension $d$, and $\mathcal{H}(\Lambda)$ the corresponding FKS conformal field theory (physically this represents the propagation of a bosonic string on the torus $\left.\mathbf{R}^{d} / \Lambda\right)$ - constructed by the action of a set of bosonic creation and annihilation operators, $a_{n}^{i}, 1 \leq i \leq d, n \in \mathbf{Z}$, satisfying canonical commutation relations, on momentum states $|\lambda\rangle, \lambda \in \Lambda$. Such theories admit an involution given by lifting the reflection symmetry of the lattice to the conformal field theory. Let us write $\mathcal{H}(\Lambda)=\mathcal{H}(\Lambda)_{+} \oplus \mathcal{H}(\Lambda)_{-}$, where $\mathcal{H}(\Lambda)_{+}$is the sub-theory invariant under this automorphism. In [2], a space $\mathcal{H}_{T}(\Lambda)$ (and a set of vertex operators acting upon it) is constructed by acting with half-integrally graded bosonic creation and annihilation operators upon a ground state forming a representation for a gamma matrix algebra related to the lattice. This forms (when $d$ is a multiple of 8) a non-meromorphic representation of $\mathcal{H}(\Lambda)$ to which the involution on $\mathcal{H}(\Lambda)$ lifts. Projecting out by this involution, we obtain a meromorphic representation $\mathcal{H}_{T}(\Lambda)_{+}$of $\mathcal{H}(\Lambda)_{+}$. It is further demonstrated in [2] that $\widetilde{\mathcal{H}}(\Lambda) \equiv \mathcal{H}(\Lambda)_{+} \oplus \mathcal{H}_{T}(\Lambda)_{+}$may be given a consistent structure as a conformal field theory (provided that $\sqrt{2} \Lambda^{*}$ is an even lattice). Now, a conformal field theory is said to be self-dual if the partition function is modular invariant (at least up to a phase under $T: \tau \mapsto \tau+1$ ), and it can be shown that $\mathcal{H}(\Lambda)$ and $\widetilde{\mathcal{H}}(\Lambda)$ are self-dual if and only if $\Lambda$ is self-dual. These are so far the only self-dual theories which have been explicitly constructed.

In view of the analogies begun in [7] and developed in [3] between codes, lattices and conformal field theories, this definition of the self-duality of a conformal field theory is rather unsatisfactory. We would prefer to say that a theory is self-dual if and only if it forms its own unique meromorphic representation [0]. We now show that these two 
definitions are equivalent in the case of the theories $\widetilde{\mathcal{H}}(\Lambda)$ (the result for $\mathcal{H}(\Lambda)$ has already been established in [8]).

Suppose that we are given a meromorphic representation $U$ of $\mathcal{H}(\Lambda)_{+}$. We construct a corresponding state $\left\langle P_{U}(z)\right|$ in $\mathcal{H}(\Lambda)_{+}$as at the beginning of section 2. The properties satisfied by this state as a consequence of it defining a representation are such that it also defines a representation of $\mathcal{H}(\Lambda)$. In general, (28-30) (or (28-29) and (31-32)) are such that any representation of a sub-theory of a conformal field theory may be extended in this way to a representation of the larger theory provided the Virasoro operators $L_{0}$ and $L_{ \pm 1}$ are identical in both theories. In particular, we may extend any representation of a sub-conformal field theory [1] to one of the full theory.

However, there is no need for the representation of $\mathcal{H}(\Lambda)$ which we have so produced to be meromorphic. All that we require is that we may restrict it to a meromorphic representation of $\mathcal{H}(\Lambda)_{+}$(specifically $U$ ). The twisted non-meromorphic representations of $\mathcal{H}(\Lambda)$ (i.e. those non-meromorphic representations such that $U\left(a_{-1}^{i}|0\rangle, e^{2 \pi i} z\right)=\omega U\left(a_{-1}^{i}|0\rangle, z\right)$ for some constant phase $\omega$ for the weight one states $a_{-1}^{i}|0\rangle$ corresponding to the Cartan subalgebra) are easily classified 国. Note however that these may not correspond to the full set of non-meromorphic representations, though they are all that we require here. They are simply either cosets of $\mathcal{H}(\Lambda)$ in $\mathcal{H}\left(\Lambda^{*}\right)$ or are built up by the action of a set of bosonic creation and annihilation operators graded by $\mathbf{Z}+r(0<r<1)$ on a ground state representing a gamma matrix algebra related to $\Lambda$. We require that the representation restrict to a meromorphic representation of $\mathcal{H}(\Lambda)_{+}$. It is clear from the fact that $L_{-1}$ generates translations, i.e. that $\left[L_{-1}, U(\psi, z)\right]=\frac{d}{d z} U(\psi, z)$, that any non-meromorphic representation of $\mathcal{H}(\Lambda)$ must be such that $U\left(a_{-1}^{i}|0\rangle, e^{2 \pi i} z\right)=\omega^{i} U\left(a_{-1}^{i}|0\rangle, z\right)$, for some phases $\omega^{i}$. Then the operator product expansion [0]

$$
U\left(a_{-1}^{i}|0\rangle, z\right) U\left(a_{-1}^{j}|0\rangle, w\right)=\delta^{i j}(z-w)^{-2}+U\left(a_{-1}^{i} a_{-1}^{j}|0\rangle, w\right)+O(z-w)
$$

implies that either $\omega^{i}=1$ for all $i$ or $\omega^{i}=-1$ for all $i$, since $U\left(a_{-1}^{i} a_{-1}^{j}|0\rangle, w\right)$ is required to be meromorphic. Hence we must have either $\mathcal{H}(\Sigma)$, for cosets $\Sigma$ of $\Lambda$ in $\Lambda^{*}$, or $\mathcal{H}_{T}(\Lambda)$ (with inequivalent representations being determined by inequivalent representations of the relevant gamma matrix algebra - there being a unique such representation only for $\Lambda$ self-dual), and so $U$ is either $\mathcal{H}(\Sigma)_{ \pm}$or $\mathcal{H}_{T}(\Lambda)_{+}$.

If we consider an irreducible representation of $\widetilde{\mathcal{H}}(\Lambda)$, then in particular it must decompose into irreducible representations of $\mathcal{H}(\Lambda)_{+}$. Considering the action of $\mathcal{H}_{T}(\Lambda)_{+}$, we then see from the list of possibilities above that the only solution when $\Lambda$ is self-dual is 
$\mathcal{H}(\Lambda)_{+} \oplus \mathcal{H}_{T}(\Lambda)_{+}$(and that there are more solutions when $\Lambda$ is not self-dual). Thus we have shown that $\widetilde{\mathcal{H}}(\Lambda)$ is truly self-dual if and only if $\Lambda$ is self-dual.

\section{Comments and Conclusions}

There is clearly much more to be done with regard to this perspective on the representation theory of bosonic meromorphic conformal field theories. In particular, we would wish to classify in some way all solutions of the equations (28-30) (and thus give a classification of the representations) for some class of conformal field theories. However, the restrictions on the state $\chi$ (i.e. that it be real and quasi-primary) are not sufficient to specify $\left\langle P\left(z^{*}\right)\right|$ uniquely, and we either have to identify some sort of cohomological equivalence classes into which the distinct states group or restrict $\chi$ further, e.g. by imposing that it be a ground state of the representation (annihilated by all positive modes of the vertex operators in the representation space). This is the subject of ongoing research. While this paper was in preparation, work of Zhu [10] came to the attention of the author in which related techniques are employed. Zhu demonstrates that the meromorphic representations of a vertex operator algebra $V$ are in one-to-one correspondence with representations of a certain algebra $A(V)$ which he constructs, though his approach is difficult to employ in practise since the algebra $A(V)$ is rather tricky to construct, and no consideration is given to non-meromorphic representations. However, Zhu effectively assumes that the state $\chi$ in our notation is a highest weight state in the representation, and shows that this means that it is annihilated by the action of the zero modes of the vertex operators corresponding to states in what he denotes by $O(V)$. This should allow us to restrict our state $\langle P(1)|$ further $(\langle P(1)|$ must be orthogonal to $O(V))$ and the consequences of this in specific examples form the subject of current research.

In addition, a general proof of the equivalence of the distinct definitions of self-duality needs to be obtained, rather than having to establish this result separately for each class of theories considered.

We note that the technique of extending a meromorphic representation to a nonmeromorphic representation of a larger conformal field theory helps us make more sense of a general abstract definition of an orbifold, as opposed to the usual geometric interpretation in terms of strings propagating on singular manifolds. Given an automorphism $\theta$ of a conformal field theory and a representation of the $\theta=1$ subspace, we may extend this representation to one of the full conformal field theory (the Virasoro modes are invariant under the action of the automorphism [8], and so the conditions required for the extension by the method of the last section to exist are therefore met). We require 
$U\left(\psi, e^{2 \pi i} z\right)=e^{\frac{2 \pi i r}{n}} U(\psi, z)$ for $\theta \psi=e^{\frac{2 \pi i r}{n}} \psi$ for consistency with the definition of an orbifold. Thus, we are able to say how a particular meromorphic representation corresponds to a certain non-meromorphic larger structure. Note though that we still have no means of showing that the orbifold is unique, beyond actually demonstrating uniqueness of solutions to the representation equations (2830) in a certain equivalence class. It should be noted that to actually verify that the orbifold is consistent is still an intricate problem which is not made any simpler in this approach. For example, in the case of $\widetilde{\mathcal{H}}(\Lambda)$, we can easily show that a $\left\langle P\left(z^{*}\right)\right|$ corresponding to $\mathcal{H}_{T}(\Lambda)_{+}$gives us a consistent representation of $\mathcal{H}(\Lambda)_{+}$, but to verify the final locality relation for consistency of $\widetilde{\mathcal{H}}(\Lambda)$ [2] requires a detailed check on matrix elements of the form

$$
\left\langle P\left(z^{*}\right)\left|V\left(\psi_{1}, z_{1}\right) \ldots V\left(\psi_{n}, z_{n}\right)\right| P\left(w^{*}\right)\right\rangle
$$

Since we find that $\sqrt{2} \Lambda^{*}$ must be even in order that this locality relation holds [2], then it is not easy to see how there can be any simple requirement on $\left\langle P\left(z^{*}\right)\right|$. Nevertheless, we feel that this point of view provides a firm foundation on which to consider both orbifolds and representations of conformal field theories from a more abstract perspective, less constrained by any requirement for an explicit construction.

\section{Acknowledgements}

The author is grateful to Gonville and Caius College, Cambridge, for a Research Fellowship and to the Royal Society for a Commonwealth Fellowship at the University of Adelaide. He would also like to thank Peter Goddard for useful conversations and encouragement. 


\section{References}

[1] L. Dolan, P. Goddard, and P. Montague. Conformal field theories, representations and lattice constructions. Submitted for publication to Communications in Mathematical Physics, hep-th/9410029.

[2] L. Dolan, P. Goddard, and P. Montague. Conformal field theory of twisted vertex operators. Nucl. Phys. B338 (1990) 529.

[3] L. Dolan, P. Goddard, and P. Montague. Conformal field theory, triality and the Monster group. Phys. Lett. B236 (1990) 165.

[4] C. Dong. Twisted modules for vertex algebras associated with even lattices. J. ALgebra 165 (1994) 91-112.

[5] I. Frenkel, J. Lepowsky, and A. Meurman. Vertex Operator Algebras and the Monster. Academic Press, New York, 1988.

[6] I.B. Frenkel, Y.-Z. Huang, and J. Lepowsky. On axiomatic approaches to vertex operator algebras and modules. 1989.

[7] P. Goddard. Meromorphic conformal field theory. In Infinite dimensional Lie algebras and Lie groups: Proceedings of the CIRM-Luminy Conference, page 556, Singapore, 1989. World Scientific.

[8] P.S. Montague. Codes, Lattices and Conformal Field Theories. PhD thesis, Cambridge University, 1991.

[9] A.N. Schellekens. Classification of ten-dimensional heterotic strings. Phys. Lett. B277 (1992) 277.

[10] Y. Zhu. Vertex operator algebras, elliptic functions, and modular forms. 1990. 